\title{
Pest Management by Nanotechnology
}

\author{
Savita Rani and Sushil ${ }^{*}$
}

Pesticide Residue Laboratory, Department of Entomology, CCS HAU, Hisar, Haryana, India

*Corresponding author

Keywords

Pest,

Nanotechnology,

Pesticide, Efficacy

Article Info

Accepted:

26 February 2018

Available Online:

10 March 2018

\section{A B S T R A C T}

Nanopesticide products symbolize a budding technical improvement that, in relation to pesticide use, could tender a variety of profits including amplified efficacy, durability and a decline in the amounts of active ingredients that require to be used. A number of formulation types have been recommended including nanoemulsions, nanocapsules and products containing immaculate engineered nanoparticles, like metals, metal oxides, and nanoclays. Nanoparticles present possibilities for more well-organized and effective control of pests, but our relative lack of information on how they act and how they can be contained are giving regulators pause before allowing their release into the environment. In the current review, we explored the type of nano-pesticides, advantages, development of nanopesticides and their future possibilities.

\section{Introduction}

A chorus of disapproval is exhibited against the use of conventional pesticide formulations because of their hazardous effect. To reduce environmental contamination and exposure to human body, new type of formulations has been developed which directly approach towards successful appliance with proper efficacy. One of the most capable is the use of nanotechnology which sponsors a highly proficient grouping of the active compound in a medium in respect to guard the core materials from undesirable reactions. Noteworthy differences may be found between nanotechnology based formulations i.e. nanoformulations and conventional formulations, principally due to magnitude and surface characteristics. Nanoparticles express possibilities for much well organised and efficient control of pests but due to short of knowledge regarding their action and practically. Useful for farmers, there is a regulatory break before their discharge into the environment.

Some nano pesticides have already been prevalent in market for many years. "Nanopesticide" does not represent a single category rather they cover numerous active products. Nanopesticides are consists of organic and inorganic constituents in different 
forms. They grip security for minimising environmental footprints left by usual formulations used in previous decades which aim for increasing solubilisation of active ingredient and its release in deliberate manner without any untimely degeneration. Term "Nanopesticide" defines for any formulation that comprises nano size (in $\mathrm{nm}$ ) elements. Nano materials like carbon nano tubes and metal oxide nanoparticles are highly efficient for the improvement of plant's germination and growth processes. The superior activities of nanopesticides will undoubtly result in new benefits and challenges to human as well as environmental health. Therefore, as present scenario is concerned, use of nanomaterials in the field of agricultural pesticide is fresh and needs further exploration. As a sum up of previous literature this article elaborates the development of ideal nanomaterials in agriculture.

Preparation of controlled release formulations (CRFs) for agriculture

\section{Types of nanoparticles used}

The largely admired shapes of nanomaterials that have been using in CRFs for biocides release are:

Nanospheres: collection in which the active compound is consistently dispersed into the polymeric template

Nanocapsules: collection in which the active compound is intense near the center core, lined by the matrix polymer

Nanogels: cross-linked polymers which can absorb large amount of water due to its hydrophilic nature.

Micelles: collection formed in aqueous solutions by molecules containing hydrophobic and hydrophilic moieties (Fig. 1).

\section{Techniques used for CRFs preparations}

\section{Chemical bonding}

This technique involves attachment of an active ingredient to a monomer and obtained a new polymer. Active ingredient are different chemical group like carboxylic acid, carbonyl group, amines which further reacts with reagents and get attached to monomer unit or polymer as such. Attachment depends upon degree of substitution, $\mathrm{pH}$ conditions of medium, particle size. Modifications of both linear as well as cross linked poly (glycidyl methacrylate) was done by previous workers (Roy et al., 2014) to prepare polymeric formulations having halo aromatic structures.

\section{Matrix capsulation}

Nanoencapsulation is a process through which a chemical is slowly but efficiently freed to the specific host for insect pest regulation. This method is most commonly used. Cyclic acetal molecules integrated in a polyamide were studied by Mosurkal et al., 2007). Gradual hydrolysis of acetal produced benzaldehyde as an active ingredient which was further studied using spectroscopic techniques. Thus chemical moiety having insecticidal property/anti-fungal activity can be incorporated for controlled release.

\section{Microencapsulation}

This approach involves a coating of small capsules smaller than 1micrometer as nanoparticles and larger than 1000 micrometer as mocro capsules. Ptea et al., (2010) demonstrated budding use of nano encapsulated polymer, which included insect growth regulators, herbicides, weedicides, insect repellents and microbial inhibitors. Zang and others (2006) characterized hydrophilic and lipophilic diamines which had chloropyriphos core. These nanoformulations 
were found to be stable at high temperature. Yang and Pan encorporated microencapsulated pesticide with urea formaldehyde polymer and got a patent for the method used. Brown et al., (2002) studied urea-formaldehyde resins with different encapsulation approaches and also examined their physical properties along with thermal resistivity.

\section{Case studies for various nanoformulations}

Calcium alginate - starch microspheres were planned using $\mathrm{CaCl}_{2}$ as cross linker (Roy et al., 2009). They were loaded with an insecticide, chlorpyriphos and both the unloaded and loaded microspheres were interpreted by FTIR and SEM to recognize the structure and morphology (Fig. 2).

Glenn et al., (2010) studied essential oil encapsulation in porous microspheres comparable in size to pollen. The microspheres were made by pumping a gelatinous aqueous 8\% high-amylose starch dissolved through an atomizing nozzle. The atomized droplets were air-classified into two fractions and collected in ethanol.

Experiments on a series of nanoemulsions of neem oil showed that the $\mathrm{LC}_{50}$ (the concentration required to achieve 50\% mortality) decreased with droplet size, which was interpreted as indicating an increased uptake of smaller droplets (Anjali et al., 2012).

A recent study has also reported the release profile of a nanoformulation in soil (Sarkar $e t$ al., 2012). A commercial formulation and nanoformulation of thiamethoxam were wrapped in parchment paper and incubated for 30 days in a sandy loam. Soil samples were regularly extracted with organic solvent to determine the amount of active ingredient transferred to the soil.
Hellman et al., (2011) discussed the advantages and drawbacks of two possible strategies for the future development of electrospun nanofibers. The first was to use high strength polymers e.g. polyamides, which do not decompose during the growth period. The second strategy was to use biologicallybased e.g., cellulose derivatives or biodegradable polymers, with the possible disadvantage of exposing non-target organisms (including human bystanders).

Nanopesticides prepared according to (Gopal et al., 2011) of fungicides and insecticides, and compared their efficacy with the conventional products. Nano-hexaconazole was characterized by SEM, TEM, and FT-IR etc. and it was found to be less than $100 \mathrm{~nm}$ in size. Patent application on Nano-hexaconazole has been filed.

Jerobin et al., (2012) prepared particles of alginate and evaluated the effects of coating agents (starch and PEG), as well as the release profile and cytotoxicity of the active agent. Different quantities of azadirachtin were encapsulated in alginate particles, employing glutaraldehyde as the reticulation agent.

The average encapsulation efficiency was $80 \%$, and was lower when the coating agents were used. Microscopy analysis showed that the particles possessed spherical morphology, and infrared analyses revealed interactions between the active agent and the components of the particles. It was concluded that the systems could be used in medicine, agriculture, and aquaculture, as well as in other areas.

Kim and Lee (2014) investigated the activity of two commercial essential oils basil (10 mg) [Ocimum basilicum (Lamiaceae)] and orange (10 mg) [Citrus sinensis (Rutaceae)] against two grain storage pests: Grain weevil and Red flour beetle [Sitophilus zeamais 
(Curculionidae)] and [Tribolium castaneum (Tenebrionidae)]. Both oils were shown to be able to be used as insecticides or fumigation agents in the management of these pests.

Bhagat et al., (2013) proposed the immobilization of pheromones within a nanogel (without using any potentially toxic chemicals such as crosslinkers or antioxidants). Evaporation of the pheromones in the nanogel was significantly reduced compared to the evaporation of the pure active gradient (AI), extending their effectiveness for up to 33 weeks compared to only three weeks for the pure AI.

\section{Pesticide residue detection by nanosensors}

To determine the pesticide residues, currently researchers follows single- and multi-residue methods based GC/LC-MS to evaluate "organophosphates, organochlorines, carbamates, triazines, triazoles, pyrethroids, neonicotinyls, strobilurins" residues in different agricultural commodities but it is time consuming too. Therefore, an alternative to analytical methods could not only increase the sampling rate but also reduce the cost associated with analytical methods. Nanosensors for pesticide residue detection offer, "high sensitivity, low detection limits, super selectivity, fast responses, and small sizes" (Liu et al., 2008).

Many workers has reported nanosensors aimed to detect the pesticide residues such as methyl parathion (Kang et al., 2010), parathion ( $\mathrm{Li}$ et al., 2006; Wang and $\mathrm{Li}$, 2008), fenitrothion (Kumaravel and Chandrasekaran, 2011) and dichlorvos (Vamvakaki and Chaniotakis, 2007). Some of these biosensors used $\mathrm{C}, \mathrm{Au}$, hybrid Titanium (Ti), Au-Platinum (Pt), and nanostructured lead dioxide $\left(\mathrm{PbO}_{2}\right) / \mathrm{TiO}_{2} / \mathrm{Ti}$ to immobilize the enzymes on sensor substrate and to increase the sensor sensitivity. The nanomaterial that would have slow targeted release of the material and also indicate deficiency (e.g. color change) of the nutrients in soil would work as an advanced alert system for farmers to decide upon the dosage rate and frequency. Nanomaterials, in addition to its use for pesticide and herbicide detection, have also been applied for pesticide degradation. Yu et al., (2007) studied the potential of using nano$\mathrm{TiO} 2$ film in photocatalytic degradation of organochlorine pesticides.

They stated that the attraction of peroxide or hydroxyl radicle and electron transfer enables the photolytic degradation of pesticides on the surface of TiO2. Enzyme based biosensors for organochlorines, organophosphates, and carbamates residue detection are also effective. But this nanomaterials based sensors applicability for pesticide residue detection might be constrained due to the large number of pesticides used in agriculture production (Liu et al., 2008; Dyk and Pletschke, 2011). Random testing of samples is not possible for all pesticides due to some issues such as:

Availability of the nanomaterials sensitive to common pesticide residues

Ease of sensor fabrication techniques and instrumentation

Desired reliability and repeatability in trace level detection,

\section{Cost}

Post effects of nanomaterial exposure to the surrounding environment need to be considered.

But as a preliminary point, the regulatory institutions can use nanosensors approach to sense major residues that are enormously damaging to human being health.

Fate of nanoformulations with their 


\section{environmental concern}

The effects that a nanoformulation has on the fate of an active ingredient may be multiple and depend on the product under consideration. The possible effects of a nanoformulation on other environmental fate processes have rarely been considered. Determining the extent to which a nanoformulation will affect processes such as the transport, relocation or bioavailability of an AI is critical to the development of a robust environmental risk assessment for nanopesticides.

A great deal of research has been carried out over the past decade into inorganic (mainly metallic) engineered nanoparticles, and the state of knowledge with regard to their fate and risk assessments have been regularly reviewed (e.g., Klaine et al., 2008, 2012). In contrast, we have focused on issues specific to organic nanocarriers, which have so far been relatively rarely considered. Polymer-based nanopesticides have received the most attention to date and can serve as a useful model with which to illustrate the issues discussed in the sections below:

\section{Transport}

Sorption is a major process affecting the transport of pesticide active ingredients following their application. The only test that has been presented for sorption in soil was for a nanoformulation of paraquat, whose release in water occurred within $8 \mathrm{~h}$ (Silva et al., 2011). Batch tests were carried out on very small quantities of soil (0.01-0.05 g) over a period of $3 \mathrm{~h}$. The sorption of the nanoformulated paraquat remained low relative to that of the pure active ingredient, even after increasing the organic matter content and/or the quantity of soil. The authors concluded that the nanoformulation could greatly improve the availability of the herbicide during use, since sorption and degradation processes were reduced. The potential for enhanced transport into surface water and ground water also needs to be assessed. When assessing the transport of nanopesticides following their application, the first step should therefore be to characterize the release profiles that are likely to occur under field conditions. The extent to which an nanocarrier can affect the transport of an active ingredient can be accessed on the basis of concepts previously developed to evaluate the potential of particle-facilitated transport of contaminants (Hofmann and Wendelborn, 2007; Kretzschmar et al., 1999) and later applied to engineered nanoparticles (Hofmann and von der Kammer, 2009).

\section{Availability to target}

Polymer formulations are known from extensive research on pharmaceutical drugs to be able to increase the bioavailability of an active ingredient (Vrignaud et al., 2011). A number of nanoformulations have been shown to be more effective than either the pure active ingredients or commercial formulations (Table 1). This could possibly be the result of a higher bioavailability and increased uptake of the nanocarrier-active ingredient, compared to the active ingredient. While an increased uptake by the target organisms is desirable, it needs to be achieved without increasing the risk to non-target organisms.

The bioavailability of the nanocarrier-active ingredient certainly depends on the carrier properties and cells/organisms considered. Studies that reported increasing efficacy or toxicity subsequent to the release of the active ingredient have also suggested that the nanocarrier-active ingredient has a limited bioavailability. There is, however, as yet no information available on any investigations into the bioavailability of nanopesticides. 
Table.1 Efficacy of nanoformulations published in review of literature by Kah and Haffman (2014)

\begin{tabular}{|c|c|c|}
\hline Type and Active Ingredient & $\begin{array}{l}\text { Efficacy of the nanoformulation } \\
\text { compared to that of a commercial } \\
\text { formulation or the pure active } \\
\text { ingredients }\end{array}$ & References \\
\hline Nanoemulsion: Neem oil & $\mathrm{LC}_{50}$ decreased with droplet size & Anjali et al., (2012) \\
\hline Permethrin & $\begin{array}{l}\text { Greater efficacy than pure } \mathrm{AI} \text { against larvae } \\
\text { ( } \mathrm{LC}_{50}(24 \mathrm{~h}) 0.006 \text { and } 0.020 \mathrm{mg} / \mathrm{L} \text {, resp.). } \\
\text { Reduced effects on non-target organisms } \\
\text { (soil bacteria and plants) }\end{array}$ & Kumar et al., (2013) \\
\hline Glyphosate & $\begin{array}{l}\text { Similar or slightly greater efficacy than } \\
\text { commercial (roundup) (pot experiments, the } \\
\mathrm{EC}_{50} \text { was } 0.40 \text { and } 0.48 \mathrm{~kg} \mathrm{AI} / \mathrm{ha} \text {, resp.) }\end{array}$ & $\begin{array}{l}\text { Jiang et al., (2012); } \\
\text { Lim et al., }(2012,2013)\end{array}$ \\
\hline $\begin{array}{l}\text { Polymer based on } \\
\text { polyethylene glycol: } \\
\text { Beta-cyfluthrin }\end{array}$ & $\begin{array}{l}\text { Greater efficacy than commercial } \\
\text { formulation when evaluated over a long } \\
\text { period (e.g., insect mortality bioassays, } \mathrm{EC}_{50} \\
(30 \mathrm{~d}) \text { were } 30-45 \mathrm{mg} / \mathrm{L} \text { and } 125 \mathrm{mg} / \mathrm{L} \text {, } \\
\text { resp.) }\end{array}$ & $\begin{array}{l}\text { Loha et al., (2012); } \\
\text { Pankaj et al., (2012) }\end{array}$ \\
\hline Carbofuran & $\begin{array}{l}\text { Greater efficacy than commercial } \\
\text { formulation (pots and field trials) }\end{array}$ & Pankaj et al., (2012) \\
\hline Acephate & $\begin{array}{l}\text { Greater efficacy than commercial } \\
\text { formulation (biochemical assays, in vitro } \\
\text { and field trials) Lower toxicity than } \\
\text { commercial formulation (mice acute oral } \\
\text { toxicity and in vitro cytotoxicity) }\end{array}$ & $\begin{array}{l}\text { Choudhury et al., (2012); } \\
\text { Pradhan et al., (2013) }\end{array}$ \\
\hline $\begin{array}{l}\text { Electrospun nanofibers } \\
\text { Thiamethoxam } \\
\end{array}$ & $\begin{array}{l}\text { Efficient at } 50 \% \text { of the recommended } \\
\text { dosage ( } 9 \mathrm{~d} \text { glass house tests, whitefly) }\end{array}$ & Xiang et al., (2013) \\
\hline $\begin{array}{l}\text { Nano inorganic + organic AI } \\
\text { Chlorfenapyr }\end{array}$ & $\begin{array}{l}\text { Insecticidal activity of silica nanoparticle } \\
\text { formulation was twice as high than that of } \\
\text { microparticles (laboratory and field tests) }\end{array}$ & Song et al., (2012) \\
\hline Validamycin & $\begin{array}{l}\text { Prolonged activity compared to pure AI (in } \\
\text { vitro germicidal test) }\end{array}$ & Qian et al., (2011) \\
\hline $\begin{array}{l}\text { Inorganic nano as active } \\
\text { ingredient } \\
\text { Silica }\end{array}$ & $\begin{array}{l}\text { Efficacy at similar rates than commercial } \\
\text { diatomaceous earth for stored grains }(0.5-2 \\
\mathrm{g} / \mathrm{kg}) \text { Effects of coating and size to be } \\
\text { further investigated }\end{array}$ & $\begin{array}{l}\text { Debnath et al., (2011, } \\
\text { 2012) }\end{array}$ \\
\hline $\mathrm{TiO}_{2}$ & $\begin{array}{l}\text { Better or on par efficacy compared to } \\
\text { standard treatment (greenhouse and field } \\
\text { trials) }\end{array}$ & Paret et al., $(2013 \mathrm{a}, \mathrm{b})$ \\
\hline Copper & $\begin{array}{l}\text { Greater efficacy than } \mathrm{Cu} \text {-oxychloride (in } \\
\text { vitro suppression of bacterial blight of } \\
\text { pomegranate at } 0.2 \mathrm{mg} / \mathrm{L} \text { and } 2500-3000 \\
\text { mg/L, resp.) }\end{array}$ & Kim et al., (2012) \\
\hline Silver & $\begin{array}{l}\text { Fungicidal activity against } 18 \text { plant } \\
\text { pathogens (Petri dishes) }\end{array}$ & Mondal and Mani (2012) \\
\hline Aluminium & $\begin{array}{l}\text { Similar or greater insecticide activity than } \\
\text { most effective commercially available } \\
\text { diatomaceous earth formulation }\end{array}$ & Stadler et al., $(2010,2012)$ \\
\hline
\end{tabular}


Fig.1 Types of nano carriers
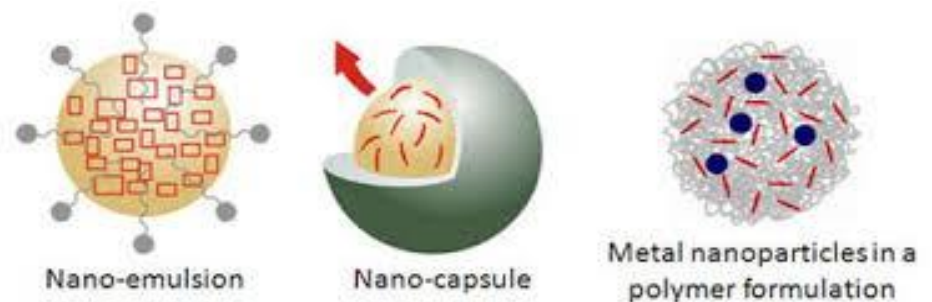

Fig.2 Commonly used biodegradable polymers incorporated with nanopesticides
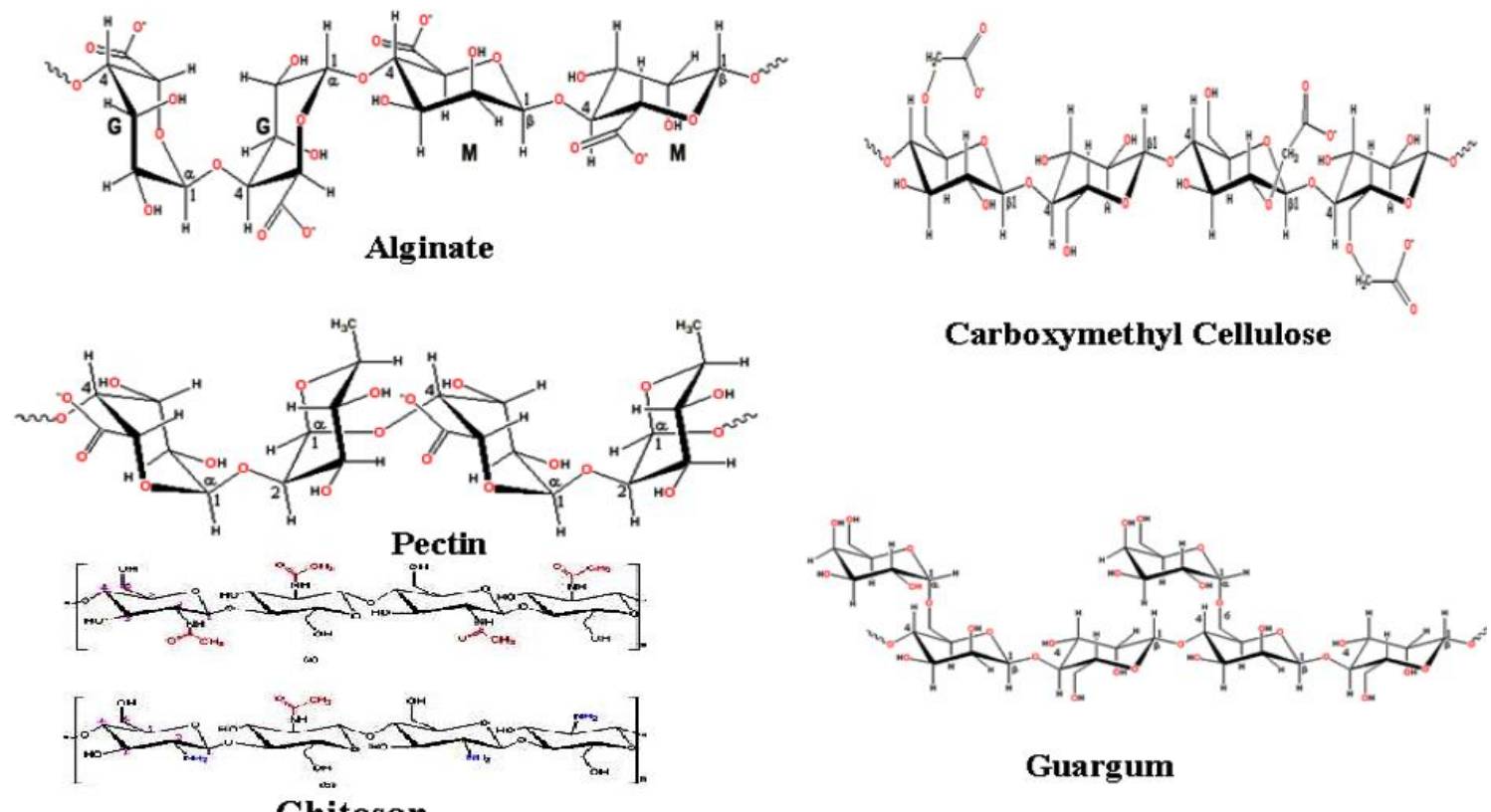

Carboxymethyl Cellulose

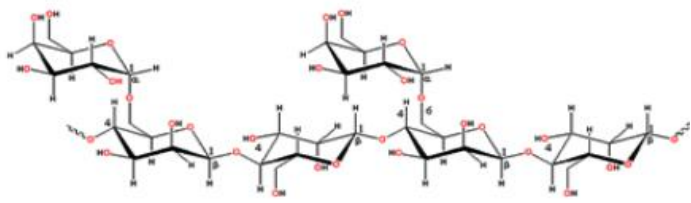

Guargum

Chitosan

Considering the active ingredient loaded onto the nanocarrier to be completely unavailable may be too simplistic, as more subtle processes may be involved. It has, for instance, been shown that chitosan (a polysaccharide frequently used as a polymer carrier for nanopesticides) can change the enantioselective bioavailability of the chiral herbicide dichlorprop (Wen et al., 2010). Qing et al., (2013) Protection of the AI against photodegradation is highly dependent on its location/distribution within the polymeric matrix. The bioavailability may likewise depend on the location of the AIs within the polymer matrix. An improved characterization of the distribution and release of the AI under a range of conditions is a prerequisite to answering the remaining key questions concerning bioavailability.

\section{Release profile}

Characterizing the durability of the nanocarrier is essential for assessing the environmental fate of nanopesticides, in both scientific and regulatory contexts. We present below a brief overview of the mechanisms and models previously developed in the pharmaceutical sector and their recent application for, and relevance to, nanopesticides. The published literature on pharmaceutical delivery systems based on 
biodegradable polymers indicates that the release mechanisms can be controlled by diffusion and/or dissolution (erosion), depending on the polymer properties, the way the active ingredient is distributed, loading and solubility. Furthermore, for a polymer matrix that is subject to swelling and dissolution (i.e., hydrophilic polymers), the thickness of the gel-layer formed will influence the diffusion pathways and thus alter the release behaviour (Kaunisto et al., 2013). The processes leading to polymer degradation are thus expected to greatly influence the release profiles. Sometimes a phase also occurs in which the remaining active ingredient is released as a result of severe degradation of the polymer matrix (Kim and Pack, 2006). In addition, if the active ingredients are not homogeneously distributed within the polymer matrixes, rapid desorption and diffusion from the surface may result in a burst of release of the active ingredients. This undesirable effect has been reported for a number of nanopesticides and has motivated the subsequent development of alternatives (e.g., nanogels or nanofibers).

\section{Hazard evaluation of nanoagrochemicals}

Hazard measurement of agricultural chemical impact on human health is not an easy process because of the great variety of substances employed, mixtures used in the field, differences in exposure dose, and geographic as well as meteorological characteristics of the agricultural areas where agro- chemicals are applied (Bolognesi, 2003; Damalas and Eleftherohotinos, 2011). Nano-specific risk assessment is a demanding subject since the assumptions used to measure the risks of straight chemicals, together with test methodologies and modelling paradigms for behavior in the environment and possible human intake may not be suitable for nanoenabled commodities (Damalas and Eleftherohotinos, 2011). The risk identification of nano-formulations requisites focus on the active ingredient concentration properties and the nano-component. A review of the body of literature on potential environmental and health hazards of nanoformulations points out the challenge of interpretation for the purpose of hazard identification (Krug, 2014). If the nanocomponent simply protects the active ingredient from degradation, then the fate and behaviour of the nano-component may be the same as in conventional pesticide formulation. Intentional and enhanced input of nanomaterials into agricultural ecosystems poses a number of questions regarding the environmental fate and transportation of these materials into the environment that still have to be answered. This seems an even more urgent issue to face considering the large number of nano-formulations potentially employed in the agricultural practice as well as the uncertainties concerning possible interactions with variable environmental factors. These may include the still unknown influence of naturally occurring ultrafine particles on the fate of nano-agrochemicals; the uncertainties concerning the alterations caused by aging, soil, and water features. However, these aspects should be taken into careful consideration because they may affect the physico-chemical characterization of nanomaterials, changing their toxicological profile and thus occupational risks. Therefore, assessing hazards posed by nanomaterials in the agricultural field may go beyond the standard strategies for assessing hazardous features from conventional chemicals or from the same nanomaterials applied in different settings. Moreover, little is known about the amount of bio solid derived nanomaterials that may enter the food webs or cause direct or indirect toxicity to plants, microbial communities, or other soil organisms, in turn affecting the ecosystem that holds nanomaterials applied for agricultural purposes, maybe influencing their 
environmental behaviour and efficacy. At hand may be potential for pesticides containing nanoscale materials to pose different risks to humans and the environment than pesticides that do not contain nanomaterials. Models generally employed for non-nano pesticides may be not appropriate for those containing nanomaterials.

As well, nano- formulations may consist of various forms of organic and/or inorganic ingredients that may also vary with time during storage and/or during/after application as a consequence of the physical, chemical, and biological processes occurring within the environment. Analytical techniques should be able to determine such changeable properties that may affect the behaviour of the nanoformulations in terms of solubility, sorption, degradation, and availability in nonequilibrium environmental compartments. Other exterior aspects touching the stability of nanomaterials - such as probable communications with tubing, medium effects, changes in $\mathrm{pH}$, the special effects of impurities, deprivation of coatings, functionalization, and/or emulsifiers that certify nanomaterial constancy may also need to be engaged while assessing work-related spotlight (Tiede et al., 2009).

\section{Future scenerio of nanoformulations}

Nanotechnology could become a potential source of emerging solutions to mitigate contamination by pesticides. The nano systems have shown great capability of controlled release pattern of active ingredient make them more efficient for long time period usability that can be solve eutrophication and residual pesticide accumulation problem. In addition, nanopesticide showed improved solubility and stabilities of active ingredient for effective control of pest (Venugopal, 2016). Still, there is need to improve the techniques for significant contribution in agricultural practices like

The current application methods need to be reviewed for increased effectiveness of nanomaterials/nanopesticides on intended targets. Lack of knowledge on the efficacy of nanopesticides remains largely unknown and further research is required before any generalizations can be made.

The current state of knowledge does not appear to be sufficient for a reliable assessment to be made of the benefits and risks associated with nanopesticides.

Studies on the harmful properties of agricultural nanochemicals should consider the possible interactions between nanosized chemicals and the multiple stressors existing in the agro-ecosystems that may result in antagonistic, synergistic, and mixture of effects, susceptibilities to environmental and health adverse effects (Handy and Shaw, 2007; Kahru and Savolainen, 2010)

Use of green chemistry and environmental sustainability principals in nanopesticide development to maximize their efficiency.

Process development for up scaling of nanopesticide for commercial level nanotechnology should promises the development of "high-tech" agricultural fields equipped with a range of intelligent.

Nanotools that allow for the precise pest management.

Applications of nanomaterials can help faster plant germination/production, effective plant protection with reduced environmental impact as opposed to traditional approaches. The strategy of the use of nanoformulations is interesting, since it can help to mitigate adverse impacts of agrochemicals on the 
environment and to the human health. As a result, there has been a substantial increase in research activity in this area. The current level of knowledge does not appear to allow a fair assessment of the advantages and disadvantages that will result from the use of some nanopesticides. In context of risk assessment, priority for research are to (i) identify the assumptions currently applied that are not valid in the case of nanopesticides, (ii) evaluate the points or situations in which differences may impact significantly on the exposure assessment outcomes, and (iii) refine or adapt current assessment protocols as required.

\section{References}

Anjali C.H., Sharma Y., Mukherjee A., Chandrasekaran N. 2012 Neem oil (Azadirachta indica) nanoemulsion as potent larvicidal agent against Culex quinquefasciatus. Pest Manag Sci. 68(2):158-63.

Bhagat D., Samanta S.K., Bhattacharya S. 2013. Efficient management of fruit pests by pheromone nanogels. Sci Rep. 3: 56-61.

Brown, E. N., Sottos, N. R. and White, S. R. 2002. Fracture testing of a self-healing polymer composite. Experimental Mechanics. 42: 372-379.

Damalas, C.A., Eleftherohorinos, I.G. 2011. Pesticide exposure, safety issues, and risk assessment indicators. Int. J. Environ. Res. Public Health. 8:14021419.

Dyk J.S.V., Pletschke B., 2011. Review on the use of enzymes for the detection of organochlorine, organophosphate and carbamate pesticides in the environment. Chemosphere. 82: 291307.

Glenn G. M., Klamczynsk A. P., Woods D. F., Chiou B. S., Orts W.J. and Imam S. H. 2010 Encapsulation of Plant Oils in
Porous Starch Microspheres J. Agric. Food Chem. 58 (7) : 4180-4184.

Gopal M., Chaudhary S.R., Ghose M., Dasgupta R., Devakumar C., Subrahmanyam B., Srivastava C., Gogoi R., Kumar R. and Goswami A. 2011. Samfungin: A novel fungicide and the process for making the same. Indian Patent Application No. 1599/DEL/2011.

Handy, R.D., Shaw, B.J., 2007. Toxic effects of nanoparticles and nanomaterials: implications for public health, risk assessment and the public perception of nanotechnology. Health Risk Soc. 9:125-144.

Hellmann C., Greiner A., Wendorff J.H. 2011. Design of pheromone releasing nanofibers for plant protection. Polym Adv Technol. 22(4):407-13.

Hofmann T, von der Kammer F. 2009. Estimating the relevance of engineered carbonaceous nanoparticle facilitated transport of hydrophobic organic contaminants in porous media. Environ Pollut. 157(4):1117-26.

Hofmann T., Wendelborn A. 2007. Colloid facilitated transport of polychlorinated dibenzo-p-dioxins and dibenzofurans (PCDD/Fs) to the groundwater at $\mathrm{Ma}$ Da area, Vietnam. Environ Sci Pollut Res. 14(4):223-4.

Jerobin J., Sureshkumar R.S., Anjali C.H., Mukherjee A., Chandrasekaran N. 2012. Biodegradable polymer based encapsulation of neem oil nanoemulsion for controlled release of Aza-A. Carbohydr Polym. 90(4):1750-6.

Kah M., Hofmann T. 2014. Nanopesticide research: Current trends and future priorities. Environment International. 63: 224-235.

Kahru, A., Savolainen, K., 2010. Potential hazard of nanoparticles: from properties to biological and environmental effects. Toxicology. 269:89-91. 
Kang T.F., Wang F., Lu L.P., Zhang Y., Liu T.S., 2010. Methyl parathion sensors based on gold nanoparticles and Nafion film modified glassy carbon electrodes. Sensor. Actuat. B-Chem. 145: 104-109.

Kaunisto E., Tajarobi F., Abrahmsen-Alami S., Larsson A., Nilsson B., Axelsson A. 2013. Mechanistic modelling of drug release from a polymer matrix using magnetic resonance microimaging. Eur J Pharm Sci. 48(4-5):698-708.

Kim K.K., Pack D.W. 2006. Chapter 2. Microspheres for drug delivery. BioMEMS and biomedical nanotechnology, vol. I. Biological and biomedical nanotechnology.

Kim S.I., Lee D.W. 2014. Toxicity of basil and orange essential oils and their components against two coleopteran stored products insect pests. $J$ Asia-Pac Entomol. 17(1):13-7.

Klaine S.J., Alvarez P.J.J., Batley G.E., Fernandes T.F., Handy R.D., Lyon D.Y. 2008. Nanomaterials in the environment: behavior, fate, bioavailability, and effects. Environ Toxicol Chem. 27(9): 1825-51.

Kretzschmar R., Borkovec M., Grolimund D., Elimelech M. 1999. Mobile subsurface colloids and their role in contaminant transport. Adv Agron. 66(66):121-93.

Krug, H.F. 2014. Nanosafety research- are we on the right track? Angew. Chem. Int. Ed. 3: 12304-12319.

Kumaravel A., Chandrasekaran M. 2011. A biocompatible nano $\mathrm{TiO} 2 /$ nafion composite modified glassy carbon electrode for the detection of fenitrothion. J. Electroanal. Chem. 650: 163-170.

Li C., Wang C., Hua S. 2006. Development of a parathion sensor based on molecularly imprinted nano-TiO2 self-assembled film electrode. Sensor. Actuat. B-Chem. 117: 166-171.
Li Q., Mahendra S., Lyon D.Y., Brunet L., Liga M.V., Li D., Alvarez P.J.J. 2008. Antimicrobial nanomaterials for water disinfection and microbial control: potential applications and implications. Water Res. 42: 4591-4602.

Liu S., Yuan L., Yue X., Zheng Z., Tang Z., 2008. Recent advances in nanosensors for organophosphate pesticide detection. Adv. Powder Technol. 19: 419-441.

Liu S., Yuan L., Yue X., Zheng Z., Tang Z., 2008. Recent advances in nanosensors for organophosphate pesticide detection. Adv. Powder Technol. 19: 419-441.

Mosurkal R., Kumar J., Parmar V.S., Watterson A.C. 2007. Controlled Release of Covalently Bound Organic Molecules by Slow Hydrolysis for Potential Biocide Application. J. of Macromol. Sci., Part A, 44: 1289.

Peteu, Serban F., Oancea, F., Sicuia O. A., Constantinescu, F., Dinu, S. 2010. Responsive Polymers for Crop Protection. Polymers. 2(3): 229-251.

Qing S., Yongli S., Yuehong Z., Ting Z., Haoyan S. Pesticide-conjugated polyacrylate nanoparticles: novel opportunities for improving the photostability of emamectin benzoate.

Roy A., Singh S.K., Bajpai J., Bajpai A.K. 2014. Controlled pesticide release from biodegradable polymers. Cent Eur J Chem. 12: 453- 469.

Roy A., Bajpai J., Bajpai A.K. 2009. Dynamics of controlled release of chlorpyrifos from swelling and eroding biopolymeric microspheres of calcium alginate and starch. Carbohydr Polym. 76:222-231.

Sarkar D.J., Kumar J., Shakil N.A., Walia S. 2012. Release kinetics of controlled release formulations of thiamethoxam employing nano-ranged amphiphilic PEG and diacid based block polymers in soil. J Environ Sci Health A Tox 
Hazard Subst Environ Eng. Nanomaterial. International Journal of 47(11):1701-12.

Nanoscience. 15(4): 1650016.

Silva Md.S., Cocenza D.S., Grillo R., de Melo N.F.S., Tonello P.S., de Oliveira L.C. 2011. Paraquat-loaded alginate/chitosan nanoparticles: preparation, characterization and soil sorption studies. J Hazard Mater. 190(1-3):36674.

Tiede, K., Hassellov, M., Breitbarth, E., Chaudhry, Q., Boxall, A.B.A. 2009. Considerations for environmental fate and ecotoxicity testing to support environmental risk assessments for engineered nanoparticles. $J$. Chromatogr. A. 1216: 503-509.

Vrignaud S., Benoit J.P., Saulnier P. 2011. Strategies for the nanoencapsulation of hydrophilic molecules in polymer-based nanoparticles.

Biomaterials. 32(33):8593-604.

Wen Y.Z., Yuan Y.L., Chen H., Xu D.M., Lin K.D., Liu W.P. 2010. Effect of chitosan on the enantioselective bioavailability of the herbicide dichlorprop to Chlorella pyrenoidosa. Environ Sci Technol. 44(13):4981-7.

Yang C.C., Pan I.H., US Patent 5576008.1996 http://www.freepatentsonline.com/5576 008.html

Vamvakaki V., Chaniotakis N.A., 2007. Pesticide detection with a liposomebased nano-biosensor. BiosensBioelectron. 22: 2848-2853.

Venugopal N.V., Sainadh N.V. 2016. Novel Polymeric Nanoformulation of Mancozeb-An Eco-Friendly

Yu B., Zeng J., Gong L., Zhang M., Zhang L., $\mathrm{Xi}$ C., 2007. Investigation of the photocatalytic degradation of organochlorine pesticides on a nanoTiO2 coated film. Talanta. 72: 16671674.

\section{How to cite this article:}

Savita Rani and Sushil. 2018. Pest Management by Nanotechnology. Int.J.Curr.Microbiol.App.Sci. 7(03): 3197-3208. doi: https://doi.org/10.20546/ijcmas.2018.703.370 